\title{
Estimation of Screw's Physical Properties Using Neural Network
}

\author{
Nan Hua Lu, ${ }^{1}$ Huang-Chu Huang, ${ }^{2}$ Shan-Jun Wu, ${ }^{1}$ and Rey-Chue Hwang ${ }^{1 *}$ \\ ${ }^{1}$ Department of Electrical Engineering, I-Shou University, \\ No. 1, Sec. 1, Syuecheng Rd., Dashu District, Kaohsiung City 84001, Taiwan \\ ${ }^{2}$ Department of Telecommunication Engineering, National Kaohsiung University of Science and Technology, \\ No. 142, Haijhuan Rd., Nanzih District, Kaohsiung City 81157, Taiwan
}

(Received December 30, 2020; accepted March 19, 2021)

Keywords: estimation, screw's physical properties, neural network, heat treatment, spheroidization

In this paper, the estimation of a screw's physical properties using a neural network $(\mathrm{NN})$ technique is presented. The aim of this research is to study the effects of various control parameters of heat treatment and spheroidization on the physical properties of an alloy steel wire in its manufacturing process. The NN model is used to analyze the data collected by the image sensor and temperature sensor for heating treatments of alloy steel wire. It is expected that an advanced screw manufacturing system with intelligent analysis ability can be developed. Then, this smart system will be able to provide the optimal control parameters in real time to produce an alloy steel wire with ideal physical properties so that high-quality screws can be produced in the later manufacturing process. The results of this study show that the NN model can indeed achieve a fairly accurate estimation of the physical properties of a steel wire after the spheroidization, quenching, and tempering heat treatments. This shows that the development of an artificial-intelligence-based screw process optimization mechanism is very feasible.

\section{Introduction}

The main function of screws is to fasten objects. In many applications such as machinery, civil engineering, construction, electronics, and automobiles, they are important and indispensable basic components. For instance, from the smallest IC chip to the largest aircraft, screws are always part of the products.

In fact, the screw industry is a traditional industry that uses little high technology, and many screw manufacturing processes still rely on the experience of senior technicians. However, with the advent of the Industry 4.0 era, reducing the reliance on the experience of senior technicians and making manufacturing equipment and control processes capable of intelligence and selfadaptive capability are the desired goals of every industry, even the traditional screw industry. The purpose of our research is to help screw companies improve their manufacturing process.

The general procedure for producing screws is shown in Fig. 1. In the production process, annealing and quenching are two procedures for changing the physical properties of steel by high-temperature heating, in which spheroidizing annealing is performed to aggregate the cementite of the steel into spheroids that are uniformly distributed in the ferrite matrix. Such an

*Corresponding author: e-mail: rchwang@isu.edu.tw https://doi.org/10.18494/SAM.2021.3243 


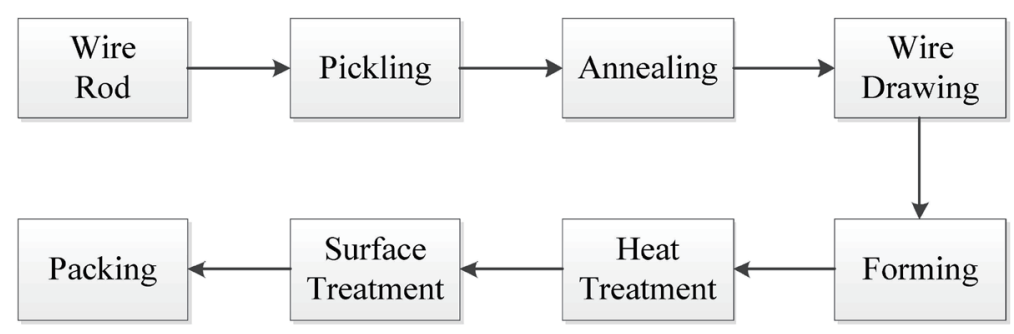

Fig. 1. Production flow chart of screws.

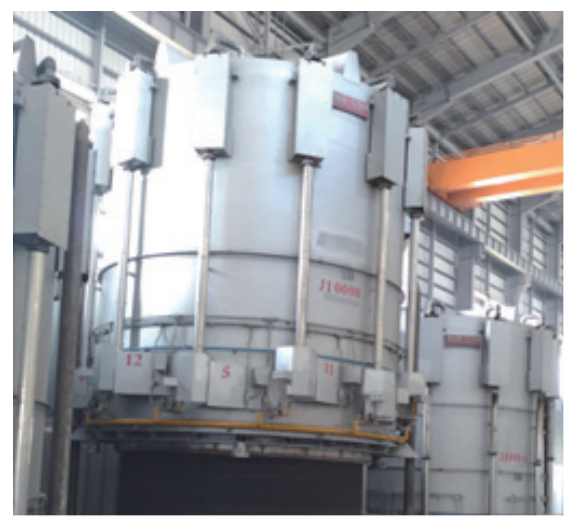

Fig. 2. (Color online) Bell-type spheroidizing annealing furnace (image provided by Fang Sheng Screw Co., Ltd.).

annealing process can improve the ductility of steel and reduce its hardness so that it can be easily machined or deformed. Figure 2 shows a bell-type spheroidizing annealing furnace, which is the main equipment used for the spheroidization.

In the spheroidization process, a thermocouple sensor is used to record the temperature changes. Figure 3 shows digital monitoring displays of the spheroidizing process.

The final heat treatment involves quenching and tempering. Quenching can increase the strength and hardness of steel. After quenching, the steel becomes brittle, so it is usually tempered again to reduce its brittleness. Basically, tempering is often accompanied by quenching. The tempering can eliminate or reduce the quenching stress, stabilize the shape and size of the steel, and prevent the deformation and cracking of quenched parts. Figure 4 displays the whole process of quenching and tempering.

\section{Literature Review}

To control the physical properties and quality of steel by heat treatment, the following related studies have been performed. Yu et al. studied the effects of the holding time during both austenitizing and spheroidizing on the microstructure and mechanical properties of the highcarbon martensitic stainless steel $8 \mathrm{Cr} 13 \mathrm{MoV} .^{(1)}$ Di et al. reported a spheroidizing procedure for the eutectic carbide in a twin roll-cast M2 high-speed steel strip by annealing, quenching, and tempering. ${ }^{(2)}$ Studies on changes in the mechanical properties of steel after the spheroidizing process have also been performed. ${ }^{(3-11)}$ In a study of quenching and tempering heat treatment, Ji 


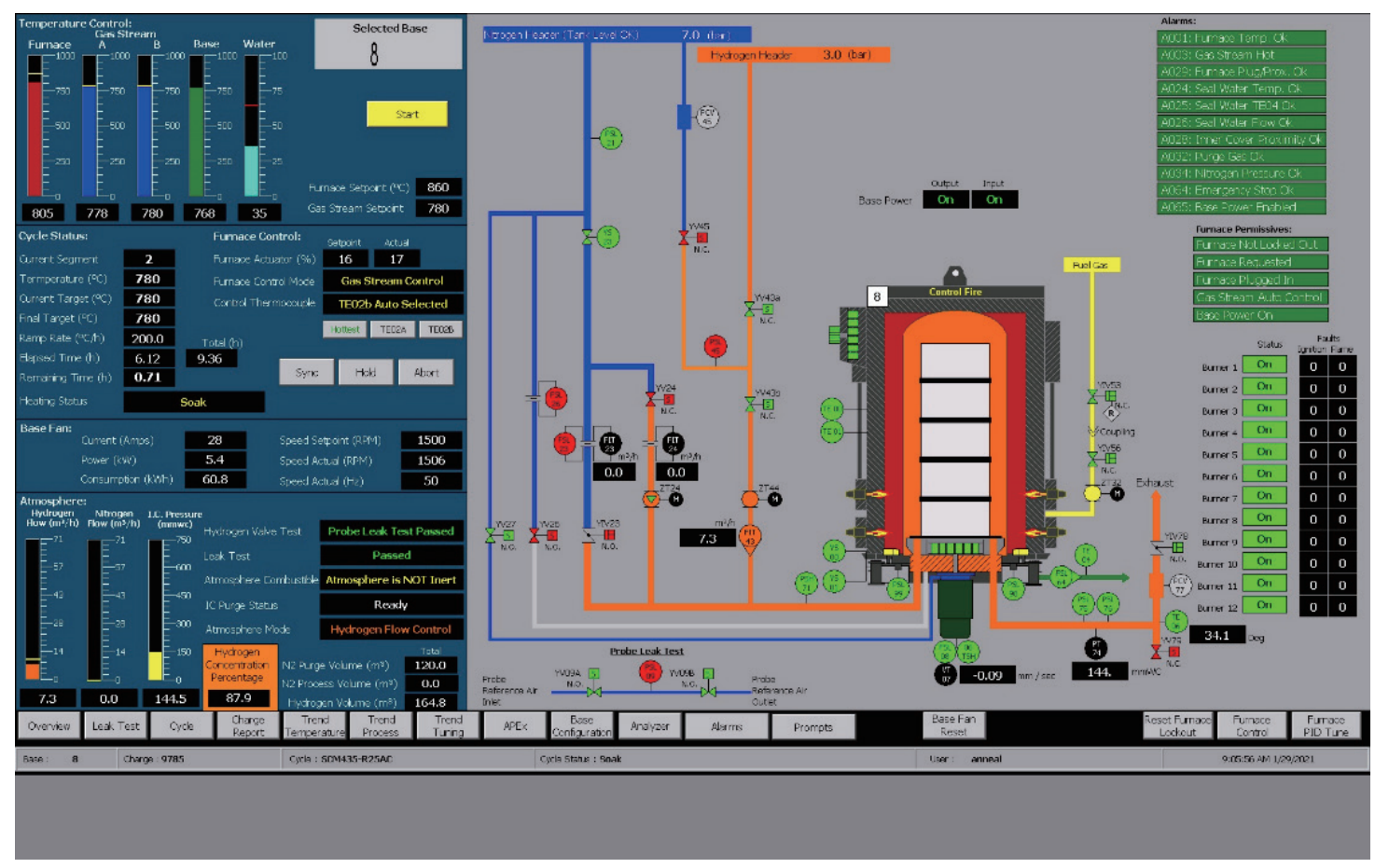

Fig. 3. (Color online) Digital monitoring displays of spheroidizing process (image provided by Fang Sheng Screw Co., Ltd.).

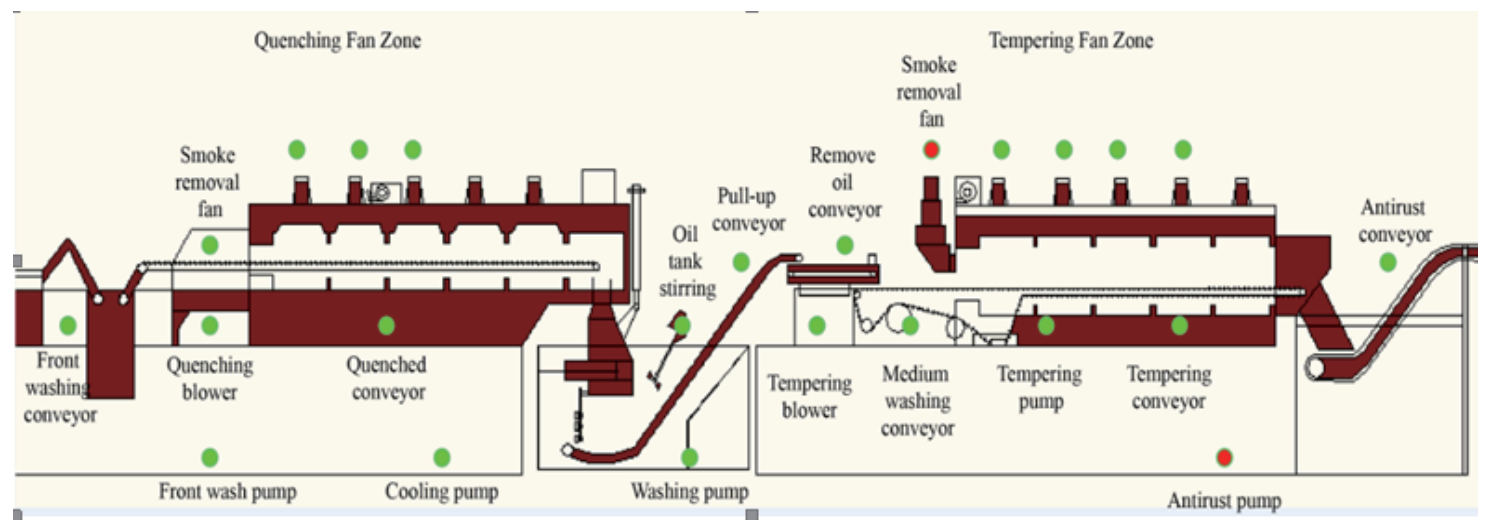

Fig. 4. (Color online) Process of quenching and tempering heat treatment (image provided by Fang Sheng Screw Co., Ltd.).

et al. used a thermal expansion analyzer, Thermo-Calc software, a scanning electron microscope, an X-ray diffractometer, and an ultrahigh-temperature confocal microscope to investigate the relationship between the microstructure and hardness of high-carbon martensitic stainless steel and temperature. ${ }^{(12-13)}$ Luo et al. studied the effect of the subcritical quenching temperature on the microstructure of an EH36 ship plate steel of $15 \mathrm{~mm}$ thickness and the performance of controlled rolling and controlled cooling (TMCP). The results show that the mechanical properties of steel can be enhanced, i.e., its hardness and plasticity can be improved. ${ }^{(14)}$ Other 
related studies on the quenching and tempering of different steels have also been reported. ${ }^{(15-21)}$

From the past research, it is known that many factors in the heat treatment affect the properties of steel wires after heat treatment. For instance, the wire diameter, wire weight, heating temperature, heating time, and cooling rate in the heat treatment could all affect the properties. However, with the large amount of production data every day, factory technicians cannot carry out troubleshooting immediately when there are problems in the manufacturing process. Usually, the relevant control can only be done in accordance with experience and by trial and error. Therefore, not only is the heating process easily prone to errors, but inappropriate heating conditions also lead to the waste of raw materials.

In our study we use artificial intelligence technology to analyze the heat treatment process. The processes of spheroidizing, quenching, and tempering were studied and performed. For the case of a variety of different screw product orders, it is hoped that artificial intelligence technology can be used to accurately set the relevant control parameters of the heating process for the different steel wire materials.

\section{Neural Network}

In this study, a neural network ( $\mathrm{NN}$ ) model was used to analyze the spheroidizing, quenching, and tempering heat treatments. Data mining is a technique that can extract hidden useful information from a large database. In data mining techniques, regression is used to obtain the relationship between the output and input variables for a system and express the output as a function of the input variables. Model specification and parameter estimation are two common applications of regression analysis.

Our research is expected to lead to the development of an estimation model for a screw's physical properties after heating treatments. Owing to its powerful learning and adaptive capabilities, the NN technique has been widely applied to the field of nonlinear regression for unknown systems. Through the training of data, a model mapping the input/output data can be automatically developed. Then, the well-trained NN can perform tasks that the user wants it to do. ${ }^{(22-25)}$

The NN structure commonly known as a multilayered feedforward network is the topology selected in this research. An example of a three-layer feedforward NN model is shown in Fig. 5. Each layer is connected to the layer above it in a feedforward manner in the sense that there is no feedback from the same layer or a layer above, and the backpropagation (BP) learning algorithm is used to train the NN model. ${ }^{(24)}$

\section{Experiments}

\subsection{Spheroidization}

In the first experiment, the hardness and spheroidizing rate of an alloy steel wire were estimated. The real hardness value of the steel wire was measured using a Rockwell hardness tester, and the spheroidizing rate was sensed with a metallographic microscope. The data 


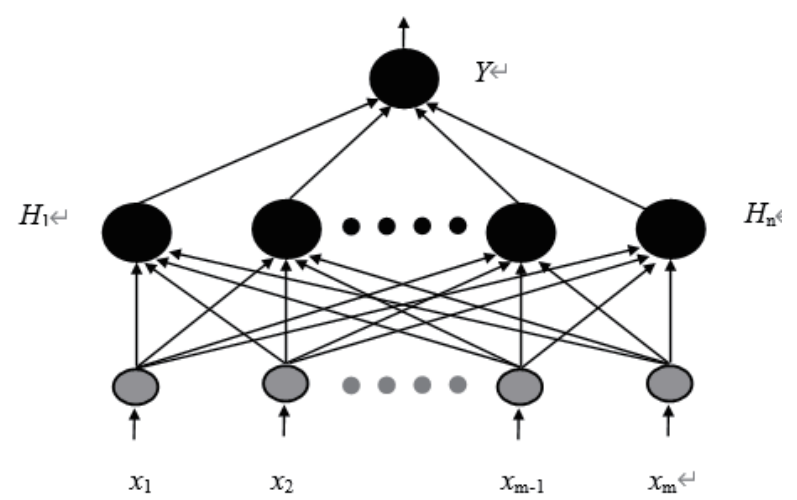

Fig. 5. Example of a three-layer NN structure.

Table 1

Number of data of each furnace for training and testing of NN.

\begin{tabular}{lccc}
\hline Furnace & Number of data & Training & Test \\
\hline A & 83 & 55 & 27 \\
B & 70 & 47 & 23 \\
C & 90 & 60 & 30 \\
\hline
\end{tabular}

collected by five spheroidizing furnaces were analyzed. All heating temperatures were sensed by a thermocouple sensor, which is a widely used type of temperature sensor. Table 1 lists the number of data of each furnace and the amount of data used for training and testing the NNs. For the estimations of the hardness and spheroidizing rate, the mean absolute percentage error (MAPE) and the accuracy are used, respectively. The size of the NN used for hardness estimation is 16-33-33-1, i.e., there are 16 nodes in the input layer, 33 nodes in the first hidden layer, 33 nodes in the second hidden layer, and one node in the output layer. The size of the NN used for spheroidizing rate estimation is 16-33-33-2. Three levels $(1,2,3)$ of the spheroidizing rate are coded as $(01,10,11)$. The inputs and outputs for both NNs are listed as follows.

Inputs: status, wire diameter, total weight, number of pieces, total spheroidizing time, five heating segment times, and five heating segment temperatures

Outputs: hardness and spheroidizing rate

Table 2 presents the estimation results of the hardness and spheroidizing rate of the three furnaces. Figures 6 and 7 respectively show the hardness estimated during training and testing of the NN for furnace A, where the solid line is the desired hardness and the dashed line is the estimated hardness.

\subsection{Quenching and tempering}

In the second experiment on quenching and tempering, the tensile strength and hardness were estimated. The values of tensile strength and hardness were respectively measured using a universal testing machine and hardness tester. The quenching and tempering temperatures were sensed by thermocouple sensors. The amounts of data collected by quenching and tempering treatments A, B, C, and D are 1346, 1008, 1113, and 2297, respectively. For each treatment, two- 
Table 2

Data for the first experiment.

\begin{tabular}{lllcc}
\hline \multirow{2}{*}{ Furnace } & \multicolumn{2}{c}{ Hardness (MAPE) (\%) } & \multicolumn{2}{c}{ Spheroidizing rate (Accuracy) (\%) } \\
\cline { 2 - 5 } & Training & Testing & Training & Testing \\
\hline A & 0.006161 & 0.876 & 100 & 96 \\
B & 0.0013 & 0.0040 & 100 & 97.77 \\
C & 0.0017 & 0.0021 & 100 & 95.55 \\
\hline
\end{tabular}

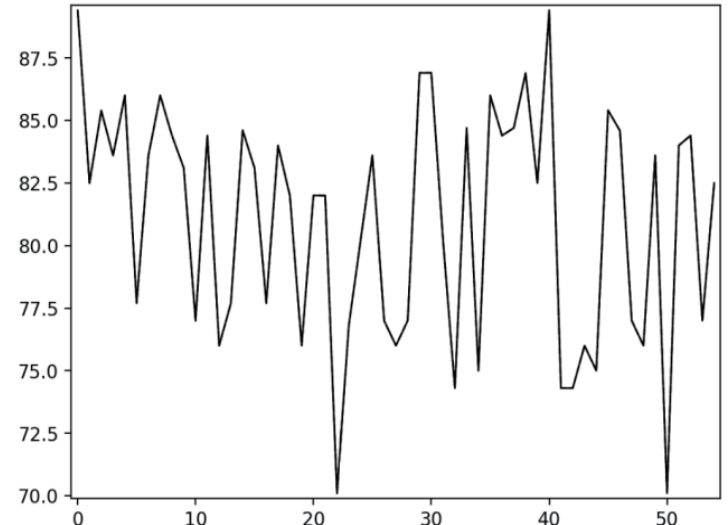

Fig. 6. Hardness during NN training.

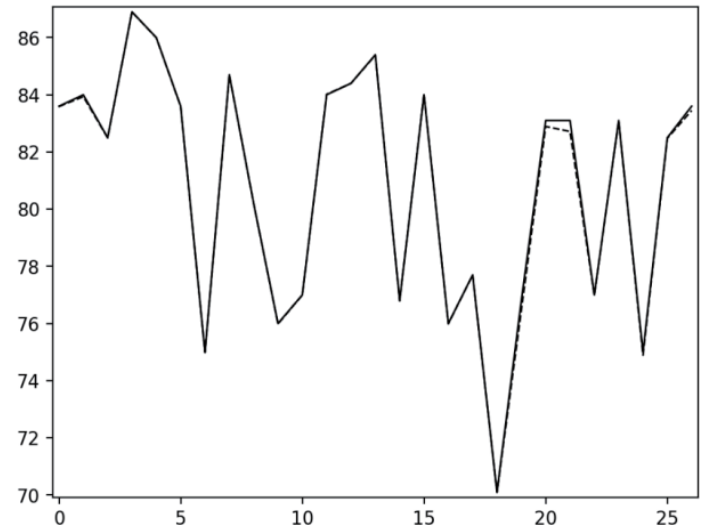

Fig. 7. Hardness during NN testing.

Table 3

Tensile strength and hardness prediction error of four treatments (A, B, C, D).

\begin{tabular}{lcccc}
\hline \multirow{2}{*}{ Treatment } & \multicolumn{2}{c}{ Tensile strength (\%) } & \multicolumn{2}{c}{ Hardness (\%) } \\
\cline { 2 - 5 } & Training (MAPE) & Test (MAPE) & Training (MAPE) & Test (MAPE) \\
\hline A & 0.695 & 1.790 & 0.833 & 1.196 \\
$\mathrm{~B}$ & 1.177 & 1.679 & 0.995 & 1.325 \\
$\mathrm{C}$ & 0.670 & 1.322 & 0.912 & 1.488 \\
$\mathrm{D}$ & 0.548 & 1.396 & 0.815 & 1.245 \\
\hline
\end{tabular}

thirds of the data are used for training the NN and the other one-third are used for testing. The size of the NN used for each treatment is 14-20-15-1, i.e., 14 nodes in the input layer, 20 nodes in the first hidden layer, 15 nodes in the second hidden layer, and one node in the output layer. The inputs and outputs of the NN for both estimations are listed as follows.

Inputs: belt speed, quenching time, two quenching temperatures, tempering time, four tempering temperatures, $\mathrm{C}, \mathrm{Mn}, \mathrm{Cr}$, and $\mathrm{Mo}$

Outputs: tensile strength and hardness

Table 3 presents the estimation results of the tensile strength and hardness for the four quenching and tempering treatments. Figures 8 and 9 respectively show examples of the estimated tensile strength for treatment A during the training and testing of the NN. Examples of the estimated hardness during the training and testing for treatment A are respectively shown in Figs. 10 and 11. Again, the solid line is the desired value and the dashed line is the estimated value. The results clearly show that the NN model can indeed estimate the screw's physical properties after the heating processes of spheroidization, quenching, and tempering. 


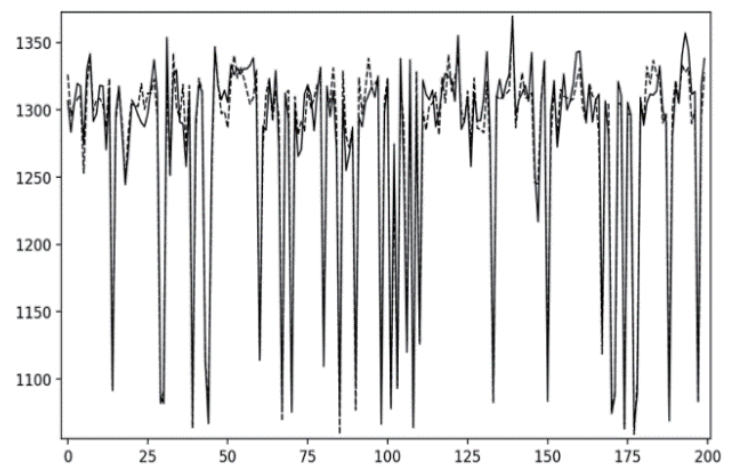

Fig. 8. Example of tensile strength estimated during training of NN.

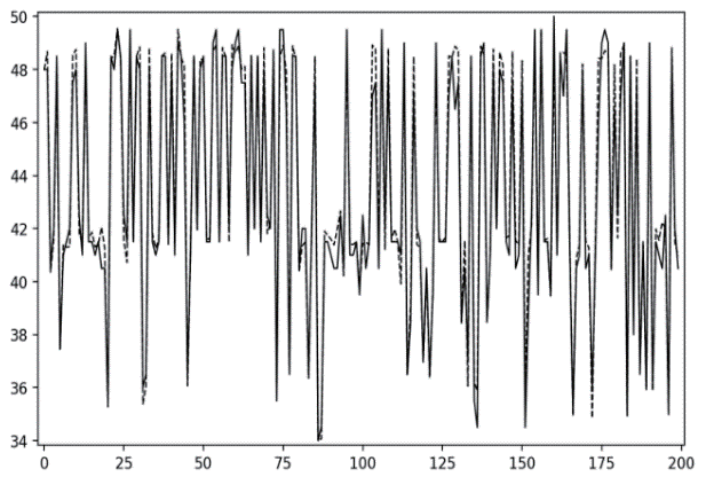

Fig. 10. Example of hardness estimated during training of $\mathrm{NN}$.

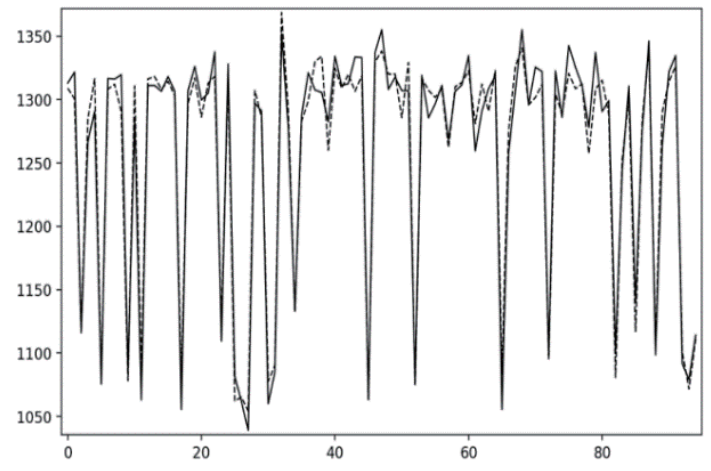

Fig. 9. Example of tensile strength estimated during testing of NN.

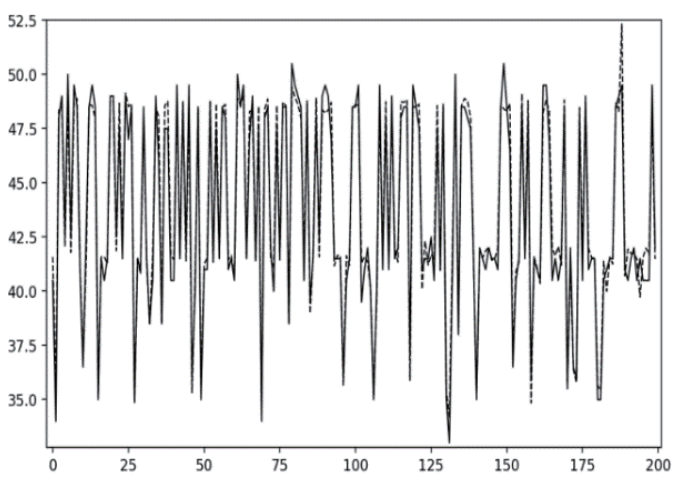

Fig. 11. Example of hardness estimated during testing of NN.

\section{Conclusions}

The main purpose of this research is to develop techniques to meet the needs of the screw industry. The NN technique is used to generate an estimation model that can predict a screw's physical properties after heating treatments. A good physical property estimation model for a screw can be used to determine appropriate control parameters in the screw manufacturing process.

In the heating treatments, temperature control and the heating time play very important roles in determining the characteristics of the screw steel. To obtain information effectively and accurately, a temperature sensor and image sensor were used to collect the relevant signal values for the learning of the $\mathrm{NN}$ and the construction of estimation modules.

Therefore, we hope that in the case of a variety of different product orders and raw materials with different chemical compositions, the relevant control parameters of heating processes can be set accurately through the use of artificial intelligence technology, enabling the production of high-quality screws. 


\section{Acknowledgments}

This research was supported by the Ministry of Science and Technology, Taiwan, under contract No. MOST-108-2221-E-214-031.

\section{References}

1 W. T. Yu, J. Li, C. B. Shi, and Q. T. Zhu: J. Mater. Eng. Perform. 26 (2017) 478. https://doi.org/10.1007/s11665016-2461-1

2 H. Di, X. Zhang, and G. Wang: J. Mater. Process. Technol. 166 (2004) 359. https://doi.org/10.1016/j. jmatprotec.2004.07.085

3 C. C. Yang and N. H. Lu: Mater. Sci. Appl. 10 (2019) 677. https://doi.org/10.4236/msa.2019.1011048

4 T. Das, J. Y. Li, M. Painter, and E. Summerville: J. Mater. Eng. Perform. 11 (2002) 86. https://doi.org/10.1007/ s11665-002-0013-3

5 Y. L. Tian and R. W. Kraft: Metall. Trans. 18 (1987) 403. https://doi.org/10.1007/BF02646654

6 K. Hono, M. Ohnuma, M. Murayama, S. Nishida, A. Yoshie, and T. Takahashi: Scr. Mater. 44 (2001) 977. https://doi.org/10.1016/S1359-6462(00)00690-4

7 J. M. O’Brien and W. F. Hosford: Metall. Mater. Trans. A 33 (2002) 1255. https://doi.org/10.1007/s11661-002$0226-\mathrm{y}$

8 D. H. Shin, S. Y. Han, K. T. Park, Y. S. Kim, and Y. N. Paik: Mater. Trans. 44 (2003) 1630. https://doi. org/10.2320/matertrans.44.1630

9 Y. G. Ko, S. Namgung, D. H. Shin, I. H. Son, K. H. Rhee, and D. L. Lee: J. Mater. Sci. 45 (2010) 4866. https:// doi.org/10.1007/s10853-010-4587-0

10 Y. P. Gul, M. A. Sobolenko, and A. V. Ivchenko: Steel Transl. 42 (2012) 531. https://doi.org/10.3103/ $\underline{\text { S0967091212060058 }}$

11 H. S. Joo, S. K. Hwang, H. M. Baek, Y. T. Im, I. H. Son, and C. M. Bae: J. Mater. Process. Technol. 216 (2015) 348. https://doi.org/10.1016/j.jmatprotec.2014.10.001

12 C. Ji, L. Wang, and M. Y. Zhu: J. Iron. Steel Res. Int. 22 (2015) 1031. https://doi.org/10.1016/S1006706X(15)30108-4

13 Q. T. Zhu, J. Li, C. B. Shi, and W. T. Yu: J. Mater. Eng. Perform. 24 (2015) 4313. https://doi.org/10.1007/s11665015-1723-7

14 X. D. Luo, Y. X. Zhu, and H. Liu: Adv. Mater. Res. 886 (2014) 59. https://doi.org/10.4028/www.scientific.net/ AMR.886.59

15 L. D. Barlow and M. Du Toit: J. Mater. Eng. Perform. 21 (2012) 1327. https://doi.org/10.1007/s11665-011-0043-9

16 A. T. Behnam, A. Fakhreddin, and H. Ali Morad: ISIJ Int. 51 (2011) 471. https://doi.org/10.2355/ isijinternational.51.471

17 M. Kianezhad and S. A. Sajjadi: Metall. Mater. Trans. A 44 (2013) 2053. https://doi.org/10.1007/s11661-0121574-x

18 S. C. Krishna, N. K. Gangwar, A. K. Jha, B. Pant, and K. M. George: J. Mater. Eng. Perform. 24 (2015) 1656. https://doi.org/10.1007/s11665-015-1431-3

19 A. Rajasekhar, G. M. Reddy, T. Mohandas, and V. S. R. Murti: Mater. Des. 30 (2009) 1612. https://doi. org/10.1016/j.matdes.2008.07.042

20 S. K. Ghosh and S. Mondal: Trans. Indian Inst. Met. 61 (2008) 33. https://doi.org/10.1007/s12666-008-0062-x

21 J. Y. Li, P. Zhao, J. Yanagimoto, S. Sugiyama, and Y. L. Chen: Int. J. Miner. Metall. Mater. 19 (2012) 511. https://doi.org/10.1007/s12613-012-0588-0

22 D. Yang, T. Jiaxin, Z. Binghong, and G. Fei: Proc. 2017 Int. Conf. Computer Systems, Electronics and Control (2018) 1081-1086. http://doi.org/10.1109/ICCSEC.2017.8446886

23 W. Z. Cui, C. C. Zhu, and H. P. Zhau: Thin Solid Films 473 (2005) 224. https://doi.org/10.1016/j.tsf.2004.06.121

24 C. Y. Shen, H. C. Huang, and R. C. Hwang: Sens. Actuators, A 147 (2008) 464. https://doi.org/10.1016/j. sna.2008.05.025

25 C. Y. Shen, H. C. Huang, and R. C. Hwang: J. Chemom. 22 (2008) 548. https://doi.org/10.1002/cem.1183 


\section{About the Authors}

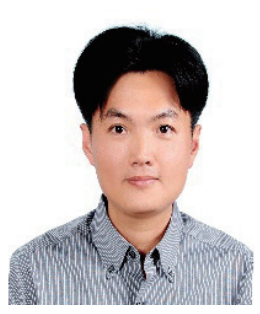

Nan Hua Lu is currently pursuing his Ph.D. degree in the Electrical Engineering Department, I-Shou University, Taiwan. His research areas include image processing, neural networks, and fuzzy theory. (nahua@livemail.tw)

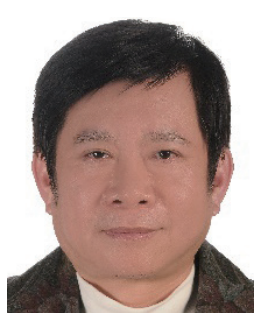

Huang-Chu Huang received his Ph.D. degree in electrical engineering from National Sun Yat-Sen University, Taiwan, in 2001. Currently, he is a professor in the Telecommunication Department, National Kaohsiung University of Science and Technology, Kaohsiung City, Taiwan. His research interests are in the areas of control, power signal prediction, and neural network applications. (h4530@nkust.edu.tw)

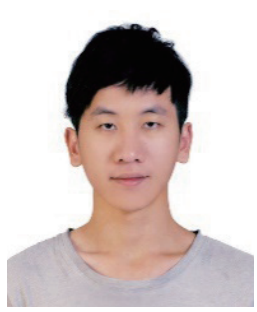

Shan-Jun Wu is currently pursuing his Master's degree in the Electrical Engineering Department, I-Shou University, Taiwan. His research interests are in neural networks and their applications. (lambertine9527@gmail.com)

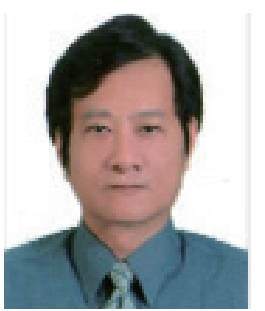

Rey-Chue Hwang received his Ph.D. degree in electrical engineering from Southern Methodist University, Dallas, TX, in 1993. Currently, he is a full professor in the Electrical Engineering Department, I-Shou University, Taiwan, R.O.C. Dr. Hwang has published more than 300 papers in various journals and conferences in the areas of artificial intelligence systems, signal processing, and fuzzy control. He is now a fellow of IET and a senior member of IEEE. He chartered the IEEE CIS Chapter, Tainan Section and served as the co-chair and chair from 2004 to 2009. (rchwang@isu.edu.tw) 